\title{
Violencia en el Noviazgo de Universitarios en México: Una Revisión
}

\section{Dating Violence in Mexico College Students: A Review}

\author{
Dr. José Luis Rojas-Solís \\ Universidad de Salamanca, España
}

\begin{abstract}
Nota sobre el Autor
José Luis Rojas-Solís. Departamento de Psicología Evolutiva y de la Educación.
\end{abstract} Facultad de Psicología, Universidad de Salamanca.

Remita cualquier duda sobre este artículo al siguiente domicilio: Departamento de Psicología Evolutiva y de la Educación. Facultad de Psicología, Universidad de Salamanca. Avda. de la Merced, 109-131, 37005. Salamanca, España. Correo electrónico:

luisrojas@usal.es, jlrojassolis@gmail.com 


\section{Resumen}

La violencia en el noviazgo en México es un problema grave porque el 76\% de los jóvenes mexicanos ha sufrido alguna vez violencia psicológica; $15 \%$, violencia física y $16.5 \%$, violencia sexual (Instituto Mexicano de la Juventud, 2008). Por ello se ha otorgado cada vez mayor atención al estudio de este fenómeno en numerosas investigaciones. Sin embargo, conocer las características de esa producción científica permitirá a consolidar o abrir nuevas líneas de investigación, por lo que esta revisión narrativa analiza las principales características metodológicas de investigaciones sobre la violencia en el noviazgo de alumnado universitario mexicano. Se incluyeron veinte estudios empíricos publicados por investigadores mexicanos y extranjeros entre 2002 y 2012, en revistas especializadas o presentadas en congresos científicos, con muestras formadas por alumnado universitario mexicano con edades entre 18 y 25 años. La búsqueda se realizó en bases de datos especializadas como Dialnet, Google Scholar, Psicodoc, Psycinfo, Redalyc y Scirus. Entre los resultados destaca que el $75 \%$ de los estudios fueron diseños cuantitativos, el $100 \%$ fue transversal y el $85 \%$ seleccionó intencionalmente la muestra. El 95\% de las muestras fueron formadas por alumnado de universidades públicas y el 15\% utilizó un cuestionario validado internacionalmente y controló la deseabilidad social de las respuestas. Se concluye la necesidad de la realización de estudios con diseños mixtos, longitudinales o diádicos con muestras seleccionadas aleatoriamente de universidades públicas y privadas. Así como la implementación de instrumentos validados que permitan contrastar los resultados obtenidos así como el control de la deseabilidad social.

Palabras clave: revisión narrativa, violencia, noviazgo, universitario, mexicano 


\begin{abstract}
Dating violence in Mexico is a serious problem because $76 \%$ of young Mexicans have suffered psychological violence, $15 \%$ have suffered physical violence and $16.5 \%$ have suffered sexual violence (Mexican Youth Institute, 2008). Therefore it has been given increasing attention to the study of this phenomenon in numerous researches. Knowing the characteristics of this production would allow to consolidate or to open up new research lines, so this narrative review discusses the main methodological characteristics of research on dating violence in Mexican university students. It was included twenty scientific researches published by Mexicans and foreigners researchers between 2002 and 2012 in scientific journals or presented at scientific conferences, with samples consisting of Mexican university students aged between 18 and 25 years. The search was conducted in specialized databases such as Dialnet, Google Scholar, Psicodoc, Psycinfo, Redalyc and Scirus. The results show that $75 \%$ of the studies were quantitative designs, $100 \%$ was cross-sectional design and $85 \%$ had nonrandom sample. $95 \%$ of the samples were formed by students from public universities and $15 \%$ used an internationally validated questionnaire and controlled social desirability of responses. It concludes the need for more studies with mixed, dyadic or longitudinal designs, but with samples randomly selected from public and private universities. The need of control of social desirability of responses and the implementation of validated questionnaires to allow comparisons between results.
\end{abstract}

Keywords: narrative review, dating violence, courtship, college student, Mexican 


\section{Violencia en el Noviazgo de Jóvenes Universitarios en México: Una Revisión}

La violencia en el noviazgo puede entenderse como“todo acto, omisión, actitud o expresión que genere, o tenga el potencial de generar daño emocional, físico o sexual a la pareja afectiva con la que se comparte una relación íntima sin convivencia ni vínculo marital" (Castro y Casique, 2010, p. 22).

El estudio sobre este fenómeno no es nada nuevo, pues hace más de cincuenta años que Kanin (1957) llamó la atención sobre el fenómeno ignorado o subestimado de la violencia en las relaciones de pareja de adolescentes y jóvenes. Algunos lustros después sería Makepeace (1981) quien con su investigación con 202 alumnos y alumnas de una universidad estatal en los Estados Unidos volvería a recuperar el interés del ámbito académico internacional sobre esta temática. A partir de este trabajo, en diversas regiones del planeta se desarrollaron innumerables estudios con adolescentes y jóvenes adultos que han constatado que la violencia en relaciones de pareja no son exclusivas de edades adultas ni de vínculos más estables como el matrimonio o la cohabitación ni teniendo como exclusiva víctima a la mujer.

Se trata de un grave problema social que trasciende fronteras por lo que México no ha sido una excepción. Por ello, en la última década la comunidad científica mexicana ha estado otorgando mayor atención al estudio de la violencia en las parejas jóvenes donde influyen muchos factores personales, familiares, ambientales o culturales (Escoto, González, Muñoz y Salomón, 2007).

Los datos más reveladores han procedido de la Encuesta Nacional de Violencia en las Relaciones de Noviazgo (ENVINOV; Instituto Mexicano de la Juventud, 2008), llevada a cabo con una muestra nacional representativa rural/urbana de 7 millones 278 mil 236 jóvenes mexicanos y mexicanas, con edades entre 15 y 24 años. El 76\% del total de la muestra de la 
ENVINOV manifestó haber sido víctima de violencia psicológica y el 15\% señaló haber experimentado al menos un incidente de violencia física; mientras que un $16.5 \%$ de las jóvenes señaló haber sufrido un evento de violencia sexual.

De esta forma, algunos estudios con jóvenes universitarios han señalado que la violencia de tipo psicológica es más frecuente si se compara con otros tipos de violencia (Olvera, Arias y Amador, 2012; Ramirez y Smithey, 2008; Valencia, García, Lozano y Flores, 2012), una violencia que no es fácilmente identificable, pero cuyos efectos también son devastadores, por ejemplo, en el rendimiento académico de las víctimas (Casanova y Russi, 2007; Sánchez y Solís, 2009).

Entre otros factores, esto habría conducido al replanteamiento de los enfoques sobre la violencia de pareja de forma que gradualmente el margen de estudio y análisis se ha comenzado a ampliar hacia más formas de violencia e incluso a la posible victimización de los hombres. Así, debido al incremento del uso de nuevas tecnologías por ambos sexos, se han comenzado a abrir líneas de investigación en la literatura internacional (Perry y Pauletti, 2011) y nacional (Trujano, Dorantes y Tovilla, 2009) debido a que estos medios electrónicos no requieren la presencia física para poder ejercer violencia, acoso o control por parte no sólo de hombres, sino también de mujeres; advirtiéndose con ello la formación de nuevos tipos de violencias y agresores.

Como resultado de las metamorfosis de la violencia de pareja se ha originado una serie de divergencias entre las investigaciones, de tal manera que, por ejemplo, la definición de la violencia que implementan muchas de ellas parece estar asociadas a ciertos "indicadores" remiten a determinadas perspectivas de abordaje.

De esta forma, por un lado se pueden encontrar aquellos trabajos que estudian la victimización de las mujeres, priorizan aspectos socioculturales y propone principalmente un 
modelo unidireccional de la violencia en la pareja que se concentra en la violencia ejercida de los hombres contra las mujeres fundamentándose en una dicotomía mujer-víctima y hombreagresor (Labrador, Fernández y Rincón, 2010). Entre otras cuestiones, sugiere que la violencia es socialmente construida y determinada principalmente por factores macroestructurales (Thompson, 1991), como la desigualdad entre hombres y mujeres (Anderson, 2005; Castro y Casique, 2010), y por ende trabajan con frecuencia con muestras femeninas analizando su victimización, pero rara vez su carácter como posibles perpetradoras de violencia, salvo por autodefensa (Dixon, Archer y Graham-Kevan, 2012).

Por otra parte, se encuentran aquellos estudios que se acercan a la violencia ejercida o cometida por ambos sexos desde un punto de vista descriptivo, podrían traslucir una perspectiva inclusiva de género (Dixon y Graham-Kevan, 2011) con un modelo multifactorial y bidireccional de la violencia. Por lo que utiliza y aplica instrumentos a hombres y mujeres por igual y atribuye la causa de la violencia a diversos factores personales, interaccionales o macroestructurales. Entre otras críticas a algunos de estos estudios podría señalarse que a veces contienen diseños ateóricos y estadísticos (Castro y Riquer, 2003).

Es así como en lo concerniente a la investigación en México actualmente conviven estudios que parten del supuesto de violencia unidireccional con investigaciones que contienen implícita o explícitamente un enfoque bidireccional de la violencia. Una situación que podría interpretarse al menos de dos formas: 1) como un cambio en el enfoque de la violencia en la pareja que incorpora nueva dimensiones del fenómeno investigado, por lo menos en la faceta de su posible bidireccionalidad, o 2) como un gradual replanteamiento del estudio psicológico de la violencia hacia una perspectiva interaccional e interpersonal, donde este fenómeno no es exclusivo de un sexo como víctima o perpetrador. 
De esta forma, parece que el enfoque bidireccional de la violencia de pareja empieza a gozar de mayor popularidad frente a los enfoques sociológicos o epidemiológicos que hasta hace unos años fundamentaban numerosos estudios sobre la violencia unidireccional en las parejas.

Unido a esta situación, es preciso señalar que en México han ocurrido numerosas transformaciones socioculturales considerando como marco el proceso de modernización vivido desde hace algunas décadas que hace posible entender fenómenos como la aparición de nuevas feminidades o masculinidades (Rojas-Solís, 2011) o modificaciones en el ámbito de las relaciones interpersonales donde pueden encontrarse las diversificaciones del noviazgo (Romo, 2008).

Es así como se ha posibilitado la eclosión de relaciones informales con gran carga afectivo-sexual que ocurren de manera espontánea, no planeada, llamadas "frees" o “amigovios" (Vizzuetth, García y Guzmán, 2010) o "amiguitos” (Weiss, 2012). Se trata de contextos relacionales donde incluso se puede evitar el término de "novios" para no implicar connotaciones de formalidad, estabilidad, compromiso o hasta obsolescencia, por lo que también se les puede denominar "salir", "andar", "novios formales", "de forajido" (sin permiso), "de manitas calientes" (sin caricias intimas o relaciones sexuales), "amantes", “calientes" (con caricias intimas y/o relaciones sexuales) (Villaseñor-Farías, 2005). De este modo, tampoco es sorprendente que el matrimonio o la convivencia permanente haya experimentado una disminución como objetivo final del noviazgo (Castro y Casique, 2010).

Teniendo en cuenta los antecedentes anteriormente descritos y debido a que no existen revisiones que se hayan centrado en las cuestiones metodológicas de las investigaciones sobre violencia en parejas de universitarios mexicanos, consideramos que existe la necesidad de contar con un panorama actualizado sobre esta temática, por lo que en este artículo nos 
proponemos contestar fundamentalmente a la pregunta: ¿cuáles son las principales características metodológicas que predominan en las investigaciones sobre violencia en parejas de universitarios mexicanos?

El objetivo central es describir cómo ha variado o, en su caso, cómo ha persistido la producción empírica nacional en función de las principales características metodológicas de algunos trabajos revisados.

La ulterior utilidad de la revisión de estos aspectos metodológicos radica principalmente en la detección de las fortalezas o debilidades de los diseños de los estudios, además de las posibles coincidencias o divergencias en los resultados como consecuencia de la metodología implementada. Cuestiones que a su vez pueden redundar en problemas que no han sido explorados, o al menos no lo suficiente, por lo que esto permitiría posteriormente entrever la consolidación o, en su caso, la apertura de nuevas líneas de investigación en la materia.

\section{Algunas consideraciones metodológicas}

Antes de continuar es preciso señalar que debido a la heterogeneidad de las fuentes consultadas, por ejemplo en el tipo de muestra o instrumentos empleados, no se usaron métodos formales de meta-análisis. Por lo tanto, es muy importante hacer énfasis en que en este artículo el abordaje del estado de la cuestión de la investigación sobre este fenómeno se realizará a través de una revisión narrativa con fines interpretativos y propositivos, más que analíticos o descriptivos.

No obstante, en aras de evitar, en la medida de lo posible, algunos de los sesgos e imprecisiones atribuidas a las revisiones narrativas (Lozano, 2005), se ha implementado una búsqueda sistemática que permita una mejor organización, descripción y análisis de los trabajos revisados. 
En ese sentido, se revisaron estudios empíricos publicados por investigadores mexicanos o extranjeros sobre la violencia en el noviazgo en universitarios mexicanos entre 2002 y 2012. En la búsqueda de la literatura se utilizaron las bases de datos: Dialnet, Google Scholar, Psicodoc, Psycinfo, Redalyc y Scirus. Las razones de su elección se fundamentaron en: 1) la posibilidad de acceso a texto completo de los estudios o a su principal información y 2) porque recogen con mayor facilidad la producción científica según los criterios de inclusión establecidos en esta revisión narrativa.

Se emplearon los descriptores en castellano: violencia, agresiones, noviazgo, universitario, mexicano; y los descriptores en inglés: dating violence, aggression, abuse, college student, Mexican.

Los criterios de inclusión fueron aquellas investigaciones originales publicadas en castellano o en inglés, en formato impreso o virtual, durante el periodo 2002-2012, que hayan sido publicadas en revistas especializadas o presentadas en congresos científicos y que tuvieran como objetivo principal el estudio de la violencia en el noviazgo con muestras formadas por alumnado universitario mexicano con edades entre 18 y 25 años.

Este rango etario se debe a: 1) la posibilidad de mayor homogeneización de los estudios, 2) porque el alumnado matriculado en estudios universitarios se ubica entre 19 y 23 años de edad, excluyendo al posgrado (Cruz y Cruz, 2008), y 3) porque puede haber personas que con 18 años ya hayan accedido a la universidad, mientras que otras pueden continuar con sus estudios con más de 23 años.

Por otra parte, se excluyeron aquellas investigaciones con muestras que no fueran exclusivamente constituidas por universitarios, las validaciones de instrumentos, las revisiones teóricas o ensayos, evaluaciones de programas preventivos, trabajos que reinterpretaran resultados de encuestas, estudios que no proveyeran suficiente información 
metodológica así como aquellos trabajos que no permitieran el acceso a los textos completos o por lo menos a los datos más relevantes de la investigación respectiva.

Por último, la organización y descripción de las investigaciones incluidas se realizó según: 1) un orden cronológico y el autor del estudio, 2) el Estado del país de donde procedía la muestra, 3) las principales características de los (as) participantes, 4) el tipo de diseño de la investigación, 5) el enfoque de violencia implementado en el estudio, 6) los instrumentos implementados y, en su caso, datos sobre su confiablidad y 7) los resultados más relevantes de la investigación respectiva.

\section{Principales características de las investigaciones sobre violencia en pareja de universitarios mexicanos realizadas el período 2002-2012}

La búsqueda de artículos referentes a la violencia en el noviazgo a través del motor de búsqueda y las bases de datos señaladas generó un total de setenta y tres resultados, de los cuales sólo veinte investigaciones cumplieron los criterios de inclusión establecidos en este artículo.

En las Tablas 1.1, 1.2 y 1.3 se muestra la descripción de algunas características de los trabajos incluidos en este artículo. 
Tabla 1.1 Principales características de las investigaciones revisadas sobre violencia en el noviazgo de jóvenes universitarios en México

\begin{tabular}{|c|c|c|c|c|c|c|}
\hline Autor (es) & Estado & Participantes & Diseño & $\begin{array}{c}\text { Enfoque de } \\
\text { violencia }\end{array}$ & $\begin{array}{l}\text { Instrumento } \\
\text { empleado }\end{array}$ & Principales resultados \\
\hline Straus (2004) & Chihuahua & $\begin{array}{l}207(\mathrm{M}) \text { y } 47 \\
\text { (V). Edad } \\
\text { promedio: } 21 \\
\text { años. }\end{array}$ & $\begin{array}{l}\text { (C), (T), } \\
\text { (SNP), } \\
\text { (MNR). }\end{array}$ & (B) & $\begin{array}{l}\text { CTS-2 } \\
\alpha>80\end{array}$ & $\begin{array}{l}\text { El } 33.3 \% \text { de los hombres, frente al } 41.2 \% \text { de las mujeres manifestaron haber agredido } \\
\text { alguna vez a su pareja. La misma tendencia se halló en el rubro de daños infligidos: } 7.9 \% \\
\text { en el caso de hombres y } 10.9 \% \text {, las mujeres. }\end{array}$ \\
\hline $\begin{array}{l}\text { Casanova y Russi } \\
(2007)\end{array}$ & Campeche & $\begin{array}{l}46 \text { (M) y } 14(\mathrm{~V}) \\
\text { Edad: } 18 \text { y } 24 \\
\text { años. }\end{array}$ & $\begin{array}{l}\text { (C), (T), } \\
\text { (SNP), } \\
\text { (MNR). }\end{array}$ & $(\mathrm{U})$ & $\alpha=(\mathrm{SI})$ & $\begin{array}{l}\text { El } 47 \% \text { de los participantes manifestó estar en relaciones con algún tipo de violencia. } \\
\text { Parece existir desconocimiento de los universitarios sobre la violencia de pareja quizá por } \\
\text { información insuficiente difundida o por un contexto sociocultural que podría mediar en la } \\
\text { identificación de la violencia. }\end{array}$ \\
\hline $\begin{array}{l}\text { Saldívar, Ramos y } \\
\text { Saltijeral (2007) }\end{array}$ & México D.F. & $\begin{array}{l}120(\mathrm{~V}) \text { y } 180 \\
(\mathrm{M}) \text {. Edad } \\
\text { promedio: } 23 \\
\text { años. }\end{array}$ & $\begin{array}{l}\text { (C), (T), } \\
\text { (SNP), } \\
\text { (MNR). }\end{array}$ & $(\mathrm{U})$ & $\begin{array}{c}* \\
\alpha>.80\end{array}$ & $\begin{array}{l}\text { Los hombres en general aceptaron más cualquier tipo de violencia como resolución de } \\
\text { conflictos, así como los mitos de violación, culpando a la mujer de su propia victimización } \\
\text { y a no creer que los hombres puedan ser violados. }\end{array}$ \\
\hline $\begin{array}{l}\text { Sánchez y Solís } \\
\text { (2007) }\end{array}$ & México D.F. & $\begin{array}{c}1036 \\
\text { universitarios. }\end{array}$ & $\begin{array}{l}(\mathrm{M}),(\mathrm{T}) \\
\text { (SP), } \\
\text { (MNR). }\end{array}$ & $(\mathrm{U})$ & $\alpha=(\mathrm{SI})$ & $\begin{array}{l}\text { Uno de cada cuatro estudiantes manifestó haber sido violentado/a alguna vez en sus } \\
\text { relaciones afectivas. La percepción de la violencia apareció como una práctica no } \\
\text { reconocida o negada. La violencia simbólica fue una práctica muy presente, pues se } \\
\text { manifiestan ideas de los celos como una expresión de amor o de que "el amor lo puede y lo } \\
\text { perdona todo". }\end{array}$ \\
\hline $\begin{array}{l}\text { Straus y Ramírez } \\
(2007)\end{array}$ & Chihuahua & $\begin{array}{l}235 \text { (M) y } 55 \\
\text { (V). Edad } \\
\text { promedio: } 19 \\
\text { años. }\end{array}$ & $\begin{array}{l}\text { (C), (T), } \\
\text { (SNP), } \\
\text { (MNR). }\end{array}$ & (B) & $\begin{array}{l}\text { CTS-2 } \\
\alpha=(\text { SI })\end{array}$ & Se halló simetría en las agresiones físicas cometidas por ambos sexos. \\
\hline $\begin{array}{l}\text { Fonseca } \quad y \\
\text { Quintero (2008) }\end{array}$ & Hidalgo & $\begin{array}{l}\text { (M) Edad: } 19 \text { a } \\
24 \text { años. }\end{array}$ & $\begin{array}{l}(\mathrm{CL}),(\mathrm{T}) \\
\text { (SNP), } \\
\text { (MNR). }\end{array}$ & $(\mathrm{U})$ & $\begin{array}{l}\text { Cuestionario } \\
\quad \text { abierto }\end{array}$ & $\begin{array}{l}\text { Estudio cualitativo que detectó la presencia de roles y estereotipos de género en las } \\
\text { participantes, de tal forma que en nombre del amor se toleran infidelidades, celos, } \\
\text { chantajes, violencia económica o agresiones sexuales, para cambiar al novio maltratador. }\end{array}$ \\
\hline $\begin{array}{l}\text { Ramirez } \\
\text { Smithey (2008) }\end{array}$ & Chihuahua & $\begin{array}{l}190(\mathrm{M}) \text { y } 30 \\
\text { (V). Edad } \\
\text { promedio: } 20 \\
\text { años. }\end{array}$ & $\begin{array}{l}\text { (C), (T), } \\
\text { (SNP), } \\
\text { (MNR). }\end{array}$ & (B) & $\begin{array}{l}\text { CTS-2 } \\
\alpha>.79\end{array}$ & $\begin{array}{l}\text { Se hallaron porcentajes similares en hombres y mujeres en cuanto a agresiones sexuales, } \\
\text { físicas y psicológicas, siendo ésta última la más común. }\end{array}$ \\
\hline
\end{tabular}

Fuente: Elaboración propia.

Nota: $(M)=$ Mujeres. $(V)=$ Hombres. $(C)=$ Cuantitativo. $(C L)=$ Cualitativo. $(M)=$ Mixto. $(T)=$ Transversal. $(\mathrm{SP})=$ Selección probabilística. $(\mathrm{SNP})=$ Selección no probabilística. $(\mathrm{MNR})=$ Muestra no representativa $(\mathrm{MR})=$ Muestra representativa. $(\mathrm{U})=$ Unidireccional. $(\mathrm{B})=$ Bidireccional. *= Instrumento elaborado ex profeso para la investigación. (SI)=Sin información. (EsEvAp)=Escala de evaluación de autoestima en la pareja. CTS-2 $=$ The revised Conflict Tactics Scales. (IFRPAA)= Identificación de factores de riesgo y protección para el autocuidado en adolescentes. (IRNU)=Inventario de Relaciones de Noviazgo en Universitarios. $(\mathrm{PVPMH})=$ Prueba de violencia de pareja en mujeres heterosexuales. $(\mathrm{RNU})=$ Relaciones de Noviazgo en Universitarios. 
Tabla 1.2 Principales características de las investigaciones revisadas sobre violencia en el noviazgo de jóvenes universitarios en México

\begin{tabular}{|c|c|c|c|c|c|c|}
\hline Autor (es) & Estado & Participantes & Diseño & $\begin{array}{l}\text { Enfoque de } \\
\text { violencia }\end{array}$ & $\begin{array}{c}\text { Instrumento } \\
\text { empleado }\end{array}$ & Principales resultados \\
\hline $\begin{array}{l}\text { Saldívar, Ramos y } \\
\text { Romero (2008) }\end{array}$ & México D.F. & $\begin{array}{l}27(\mathrm{M}) \text { y }(\mathrm{V}) \\
\text { Edad: } 18 \text { a } 25 \\
\quad \text { años. }\end{array}$ & $\begin{array}{l}\text { (CL), (T), } \\
\text { (SNP), } \\
\text { (MNR). }\end{array}$ & $(\mathrm{U})$ & Grupo focal & $\begin{array}{l}\text { Hombres y mujeres asocian la coerción sexual con el uso de una fuerza extrema; la gran } \\
\text { mayoría no tiene claro su significado. Ambos culpan a las segundas de su victimización y } \\
\text { están de acuerdo que las mujeres padecen más coerción sexual, aunque mencionan que los } \\
\text { hombres también pueden ser víctimas. }\end{array}$ \\
\hline $\begin{array}{l}\text { Lumbreras et al. } \\
\text { (2009) }\end{array}$ & Tlaxcala & $\begin{array}{l}1,649(\mathrm{M}) \mathrm{y} \\
1,010(\mathrm{~V}) . \text { Edad } \\
\text { promedio: } 20 \\
\text { años. }\end{array}$ & $\begin{array}{l}\text { (CL), (T), } \\
\text { (SP), } \\
\text { (MNR). }\end{array}$ & (U) & $\begin{array}{l}\text { Cuestionario } \\
\text { abierto }\end{array}$ & $\begin{array}{l}198 \text { mujeres manifestaron haber sufrido algún tipo de violencia; el } 38 \% \text { de ellas reportó } \\
\text { haber sufrido agresiones verbales, } 36 \% \text { agresiones físicas, } 7 \% \text { agresiones con arma y } 19 \% \text {, } \\
\text { violencia sexual. }\end{array}$ \\
\hline $\begin{array}{l}\text { Saldívar y Romero } \\
\text { (2009) }\end{array}$ & México D.F. & $\begin{array}{l}159(\mathrm{~V}) \text { y } 161 \\
(\mathrm{M}) \text {. Edad } \\
\text { promedio: } 21 \\
\text { años. }\end{array}$ & $\begin{array}{l}\text { (C), (T), } \\
\text { (SNP), } \\
\text { (MNR). }\end{array}$ & $(\mathrm{U})$ & $\begin{array}{l}\text { Cuestionario } \\
\text { abierto }\end{array}$ & $\begin{array}{l}33.4 \% \text { de la muestra ha sido víctima de coerción sexual, } 9.4 \% \text { mencionó haber ejercido } \\
\text { coerción sexual hacia su pareja. Los varones ejercieron más coerción sexual. Los y las } \\
\text { participantes señalaron que los hombres usan más tácticas indirectas mientras que las } \\
\text { mujeres utilizan más las tácticas directas. }\end{array}$ \\
\hline $\begin{array}{l}\text { Sánchez y Solís } \\
\text { (2009) }\end{array}$ & México D.F. & $\begin{array}{l}918(\mathrm{M}) \text { y }(\mathrm{V}) \\
\text { estudiantes de la } \\
\text { FES. }\end{array}$ & $\begin{array}{l}(\mathrm{C}),(\mathrm{T}) \\
(\mathrm{SNP}) \\
(\mathrm{MNR})\end{array}$ & (B) & $\alpha=(\mathrm{SI})$ & $\begin{array}{l}26 \% \text { de los varones y } 35 \% \text { de las mujeres ha experimentado violencia, uno de cada cuatro } \\
\text { estudiantes señala que las violaciones pueden ser provocadas por la forma de vestir de las } \\
\text { mujeres. La dificultad en reconocer la violencia se debió a su asociación con el maltrato } \\
\text { físico. }\end{array}$ \\
\hline $\begin{array}{l}\text { González, } \\
\text { Hernández } \\
\text { Garza (2010) }\end{array}$ & Coahuila & $\begin{array}{l}167(\mathrm{M}) \text { y } 79 \\
\text { (V). Edad: } 17 \text { a } \\
24 \text { años. }\end{array}$ & $\begin{array}{l}(\mathrm{C}),(\mathrm{T}) \\
\text { (SNP), } \\
\text { (MNR). }\end{array}$ & (B) & $\begin{array}{c}* \\
(\mathrm{IRNU}) \\
\alpha=(\mathrm{SI})\end{array}$ & $\begin{array}{l}\text { Hombres y mujeres siguen manteniendo creencias y actitudes que determinan su } \\
\text { comportamiento, así los prejuicios de género se asociaron a la frecuencia de episodios } \\
\text { violentos del sujeto hacia su pareja y de ésta hacia él. }\end{array}$ \\
\hline $\begin{array}{l}\text { Ramírez y Núñez } \\
\text { (2010) }\end{array}$ & Sonora & $\begin{array}{l}188(\mathrm{~V}) \text { y } 188 \\
(\mathrm{M}) \text {. Edad } \\
\text { promedio: } 21 \\
\quad \text { años. }\end{array}$ & $\begin{array}{l}(\mathrm{C}),(\mathrm{T}) \\
(\mathrm{SNP}) \\
(\mathrm{MR})\end{array}$ & (U) & $\begin{array}{c}* \\
\alpha=.87\end{array}$ & $\begin{array}{l}\text { Se describen diversos factores comunes en las relaciones violentas en parejas jóvenes } \\
\text { hallándose que la autoestima, los estilos de crianza, factores sociales y económicos, en ese } \\
\text { orden, estuvieron relacionados con la aparición de la violencia. Un posible factor protector } \\
\text { es un mayor nivel educativo. }\end{array}$ \\
\hline $\begin{array}{l}\text { Rangel y García } \\
(2010)\end{array}$ & $\begin{array}{l}\text { San Luis } \\
\text { Potosí }\end{array}$ & $\begin{array}{c}165(\mathrm{M}) \text { y } 85 \\
\text { (V). Edad } \\
\text { promedio: } \\
\text { 21años. }\end{array}$ & $\begin{array}{l}\text { (C), (T), } \\
\text { (SP), } \\
\text { (MNR). }\end{array}$ & (B) & $\begin{array}{c}* \\
\text { (IFRPAA) } \\
\alpha=.78\end{array}$ & $\begin{array}{l}\text { No se halló diferencia significativa en la violencia de pareja sufrida tanto por hombres } \\
\text { como mujeres. Entre otros factores, las conductas violentas se relacionaron con el hecho de } \\
\text { haber sido receptores de violencia por los padres. }\end{array}$ \\
\hline
\end{tabular}

Fuente: Elaboración propia.

Nota: $(M)=$ Mujeres. $(\mathrm{V})=$ Hombres. $(C)=$ Cuantitativo. $(\mathrm{CL})=$ Cualitativo. $(\mathrm{M})=$ Mixto. $(\mathrm{T})=$ Transversal. $(\mathrm{SP})=$ Selección probabilística. (SNP)=Selección no probabilística. $(\mathrm{MNR})=$ Muestra no representativa. $(\mathrm{MR})=$ Muestra representativa. $(\mathrm{U})=$ Unidireccional. $(\mathrm{B})=$ Bidireccional. *= Instrumento elaborado ex profeso para la investigación. (SI)=Sin información. (EsEvAp)=Escala de evaluación de autoestima en la pareja. CTS-2= The revised Conflict Tactics Scales. (IFRPAA)= Identificación de factores de riesgo y protección para el autocuidado en adolescentes. (IRNU)=Inventario de Relaciones de Noviazgo en Universitarios. (PVPMH)=Prueba de violencia de pareja en mujeres heterosexuales. (RNU)=Relaciones de Noviazgo en Universitarios. 
Tabla 1.3 Principales características de las investigaciones revisadas sobre violencia en el noviazgo de jóvenes universitarios en México

\begin{tabular}{|c|c|c|c|c|c|c|}
\hline Autor (es) & Estado & Participantes & Diseño & $\begin{array}{l}\text { Enfoque de } \\
\text { violencia }\end{array}$ & $\begin{array}{c}\text { Instrumento } \\
\text { empleado }\end{array}$ & Principales resultados \\
\hline $\begin{array}{l}\text { García y Romero } \\
\text { (2011) }\end{array}$ & Hidalgo & $\begin{array}{c}200(\mathrm{~V}) \text { y } 200 \\
\text { (M). }\end{array}$ & $\begin{array}{l}\text { (CL), (T), } \\
\text { (SNP), } \\
\text { (MNR). }\end{array}$ & (B) & $\begin{array}{c}\text { Redes } \\
\text { semánticas. }\end{array}$ & $\begin{array}{l}\text { Para hombres y mujeres la coerción sexual implica violencia emocional en términos de } \\
\text { chantajes, amenazas y manipulación, pero también implica técnicas como la seducción, los } \\
\text { besos y las caricias. En muchos de los casos parece difícil distinguir entre la seducción y el } \\
\text { chantaje para el inicio de las relaciones sexuales. }\end{array}$ \\
\hline $\begin{array}{l}\text { Torres, Carreón, } \\
\text { Sánchez, Bernal y } \\
\text { Peña (2011) }\end{array}$ & Guerrero & $\begin{array}{l}766(\mathrm{M}) . \text { Edad } \\
\text { promedio: } 21 \\
\text { años. }\end{array}$ & $\begin{array}{l}\text { (C), (T), } \\
\text { (SP), } \\
\text { (MR). }\end{array}$ & $(\mathrm{U})$ & $\begin{array}{c}* \\
\alpha=(\mathrm{SI})\end{array}$ & $\begin{array}{l}26.8 \% \text { de la muestra refirió haber sufrido violencia psicológica; } 23.2 \% \text {, física; } 7.6 \% \text {, } \\
\text { económica y } 2.6 \% \text {, sexual. Factores de riesgo asociados a la violencia fueron: consumo de } \\
\text { alcohol o drogas, violencia familiar o comunitaria. La autoestima alta se identificó como un } \\
\text { factor protector. }\end{array}$ \\
\hline $\begin{array}{l}\text { Adams } \\
\text { Cervantes (2012) }\end{array}$ & México D.F. & $400(\mathrm{M})$ & $\begin{array}{l}\text { (C), (T), } \\
\text { (SNP), } \\
\text { (MNR). }\end{array}$ & $(\mathrm{U})$ & $\begin{array}{c}* \\
(\text { EsEvAp) } \\
\alpha=.83\end{array}$ & $\begin{array}{l}\text { Se investigó la relación entre la violencia pasiva y la autoestima en dos instituciones } \\
\text { pública y privada. Las estudiantes de la universidad privada fueron más propensas a } \\
\text { establecer relaciones de violencia pasiva considerando normales conductas de control por } \\
\text { parte de la pareja y dependencia hacia la misma. }\end{array}$ \\
\hline $\begin{array}{l}\text { Oliva, González, } \\
\text { Yedra, Rivera y } \\
\text { León (2012) }\end{array}$ & Veracruz & $\begin{array}{l}899(\mathrm{~V}) \text { y } 1089 \\
\text { (M). Edad } \\
\text { promedio: } 21 \\
\quad \text { años. }\end{array}$ & $\begin{array}{l}\text { (C), (T), } \\
\text { (SP), } \\
\text { (MR). }\end{array}$ & (B) & $\begin{array}{c}* \\
(\mathrm{RNU}) \\
\alpha=.84\end{array}$ & $\begin{array}{l}\text { Se analizaron diversas formas de agresión en el noviazgo, en general no se encontró } \\
\text { diferencia significativa en la perpetración por ambos sexos salvo en algunas conductas } \\
\text { concretas asociadas a cuestiones de género. Un factor asociado a la violencia destacable fue } \\
\text { la observación de modelos violentos en la familia de origen. }\end{array}$ \\
\hline $\begin{array}{l}\text { Olvera } \\
\text { (2012) }\end{array} \quad$ et $\quad$ al. & $\begin{array}{l}\text { Estado de } \\
\text { México }\end{array}$ & $100(\mathrm{M})$ & $\begin{array}{l}\text { (C), (T), } \\
\text { (SP), } \\
\text { (MNR). }\end{array}$ & $(\mathrm{U})$ & $\begin{array}{c}* \\
(\mathrm{PVPMH}) \\
\alpha=(\mathrm{SI})\end{array}$ & $\begin{array}{l}\text { Se estudiaron la violencia económica, psicológica, física y sexual. El } 92 \% \text { de las } \\
\text { participantes señalaron haber sufrido con más frecuencia violencia psicológica que además } \\
\text { casi siempre estuvo acompañada de otro tipo de violencia, la combinación más recurrente } \\
\text { fue: violencia psicológica, económica y física. }\end{array}$ \\
\hline $\begin{array}{l}\text { Valencia } \quad \text { et } \quad \text { al. } \\
(2012)\end{array}$ & Michoacán & $\begin{array}{l}256(\mathrm{M}) \text { y } 95 \\
\text { (V). Edad } \\
\text { promedio: } 22.5 \\
\text { años. }\end{array}$ & $\begin{array}{l}\text { (C), (T), } \\
\text { (SNP), } \\
\text { (MNR). }\end{array}$ & (B) & $\begin{array}{c}* \\
\alpha=.80\end{array}$ & $\begin{array}{l}\text { La violencia psicológica fue la más frecuente, seguida de la sexual y, por último la física. } \\
\text { En los tres casos tanto hombres como mujeres señalaron haber sido perpetradores o } \\
\text { receptores de la violencia. }\end{array}$ \\
\hline
\end{tabular}

Fuente: Elaboración propia.

Nota: $(M)=$ Mujeres. $(V)=$ Hombres. $(C)=$ Cuantitativo. $(C L)=$ Cualitativo. $(M)=$ Mixto. $(T)=$ Transversal. $(S P)=$ Selección probabilística. $($ SNP $)=$ Selección no probabilística. $($ MNR $)=$ Muestra no representativa. $(\mathrm{MR})=$ Muestra representativa. $(\mathrm{U})=$ Unidireccional. $(\mathrm{B})=$ Bidireccional. *= Instrumento elaborado ex profeso para la investigación. (SI)=Sin información. (EsEvAp)=Escala de evaluación de autoestima en la pareja. CTS-2= The revised Conflict Tactics Scales. (IFRPAA)= Identificación de factores de riesgo y protección para el autocuidado en adolescentes. (IRNU)=Inventario de Relaciones de Noviazgo en Universitarios. $(\mathrm{PVPMH})=$ Prueba de violencia de pareja en mujeres heterosexuales. (RNU)=Relaciones de Noviazgo en Universitarios. 
A simple vista puede observarse que en los últimos cinco años se ha experimentado un gran crecimiento en el número de investigaciones. Sin embargo, un primer aspecto subrayable de las investigaciones es que han partido de diferentes definiciones de violencia originando así resultados diversos. Empero, se podría decir que en los trabajos revisados predomina un carácter esencialmente descriptivo de la violencia en el noviazgo, principalmente en aquellas de naturaleza psicológica, física y sexual.

El diseño de investigación de la mayoría de los estudios fue cuantitativo (75\%), transversal (100\%) y la selección de la muestra fue no probabilística (75\%). El número de investigaciones cualitativas fue menor (25\%). Sólo se halló un estudio con diseño mixto y, al momento de realizar esta revisión, no se encontró ninguna investigación de carácter longitudinal o diádica.

El 75\% del total de las investigaciones incluyó a hombres y mujeres, cuatro estudios sólo a mujeres (20\%), mientras que un estudio proveyó información incompleta sobre los participantes.

El 30\% del número total de las investigaciones se llevó a cabo con población de México D.F. y el resto se distribuyó en diferentes Estados de la República Mexicana.

El rubro de los instrumentos es muy importante en el caso de las quince investigaciones cuantitativas, pues en el $80 \%$ de ellas se elaboraron instrumentos ex profeso para el estudio respectivo.

En contraparte, sólo tres estudios realizados en Ciudad Juárez (Straus, 2004; Straus y Ramírez, 2007; Ramirez y Smithey, 2008), usaron un instrumento como el The revised Conflict Tactics Scales (CTS-2; Straus, Hamby, Boney-McCoy y Sugarman, 1996) que es ampliamente utilizado en varias partes del mundo. Pese a las bondades y 
popularidad de este instrumento, es preciso señalar que no fue diseñado específicamente para población joven (Rodríguez-Franco et al., 2010). Además, al realizarse una búsqueda específica sobre validación del CTS-2 en México sólo se halló el trabajo realizado por Mora, Natera, Tiburcio y Juárez (2008) con una muestra formada por 230 mujeres residentes en la ciudad de Pachuca, Hidalgo, pero con una edad promedio de 34 años.

Entre los factores causales o de riesgo de la violencia de pareja sobresalieron: la transmisión intergeneracional de la violencia, el consumo de sustancias o la adscripción a roles de género tradicionales.

\section{Discusión}

En primer lugar se discutirán los aspectos metodológicos de las investigaciones revisadas, acto seguido se comentarán algunos aspectos de contenido.

En ese orden de ideas, la mayoría de los estudios se concentraron en la descripción de la violencia física, psicológica o sexual, mientras que sólo un estudio tuvo fue más allá de la descripción o correlación de variables a través de un modelo predictivo mediante regresión lineal (González et al., 2010).

Esto es algo de suma importancia ya que, como Dutton (1994) indica, el problema de atribuir todo el peso predictivo o causal de la violencia a factores macrosistémicos -en el caso del enfoque unidireccional de la violencia- es que puede omitir otras variables como las individuales, diádicas, microsistémicas o exosistémicas para tener una mejor comprensión de un problema complejo. En ese sentido, además de describir el fenómeno a través de su prevalencia o la correlación de los factores asociados, sería muy conveniente conocer la capacidad explicativa de distintas variables asociadas a este fenómeno, además de las macrosistémicas. Esto sin duda requeriría del 
diseño y uso de "análisis tradicionales", como los ANOVAs o los análisis de regresión lineales, donde la unidad de análisis es la persona (Kenny y Cook, 1999), pero también de los análisis multivariados o las ecuaciones estructurales que permitan estimar el efecto y las relaciones entre múltiples variables (Ruiz, Pardo y San Martín, 2010).

Ahora bien, en el estudio de la violencia de pareja concurre una dimensión valorativa que implica problemas asociados a la percepción en la perpetración y victimización por las interpretaciones que tanto hombres como mujeres otorgan a estos comportamientos (Castro y Riquer, 2004), algo en lo que los autoinformes suelen fallar. A ese respecto, es conveniente señalar que desde la literatura internacional se ha venido enfatizando la gran necesidad de realizar estudios con diseños que combinen metodología cuantitativa y cualitativa para la identificación y valoración de la diferentes interacciones en la pareja entre las que está, por supuesto, la violencia (Wolfe, Scott y Crooks, 2005).

Con todo, sólo un estudio revisado, concretamente el de Sánchez y Solís (2007), combinó metodología cuantitativa y cualitativa. Añadido a esto, es preciso indicar la pertinencia de diseños longitudinales que suministren conocimientos acerca de aspectos que, hasta ahora, han sido poco o nada abordados como el significado de la violencia ejercida y recibida en ambos sexos, las trayectorias de violencia o los emparejamientos selectivos (Lorber y O’Leary, 2012).

Por otra parte, pese a que hace años se advirtiera de la naturaleza interaccional y diádica de los comportamientos en la pareja (Huston y Robins, 1982), la totalidad de los estudios revisados sólo incluyó un miembro de la pareja en sus diseños. Esto, sin embargo, no es algo privativo de la investigación mexicana ya que algunas investigaciones internacionales que estudian la bidireccionalidad de la violencia de 
pareja, por ejemplo la realizada por Melander, Noel y Tyler (2010), siguen elaborando sus apreciaciones basándose en la inclusión de un sólo un integrante de la díada.

Lo cierto es que la información obtenida de un miembro de la relación no necesariamente es representativa de la otra persona, dificultando así la extrapolación de los datos (Shorey, Cornelius, y Bell, 2008). Por lo tanto, existe una gran necesidad de llevar a cabo estudios diádicos en parejas jóvenes, algo hasta ahora inexistente en la literatura empírica nacional, al menos al momento de realizarse esta revisión.

En cuanto a los instrumentos utilizados es muy pertinente señalar la gran cantidad y diversidad de cuestionarios construidos ex profeso para los estudios lo que, entre otras cosas, dificulta la réplica, contrastación o refutación de sus resultados.

Caso contrario a los tres estudios (Ramirez y Smithey, 2008; Straus, 2004; Straus y Ramírez, 2007) que implementaron el CTS-2 que, por cierto, hallaron similares resultados. Aunado a ello, es muy llamativo que sólo en estos tres casos se haya controlado la variable de la deseabilidad social en las respuestas. Se trata de algo sumamente importante para los estudios cuantitativos, pues entre las críticas que sobresalen al uso de los autoinformes se encuentra la alta deseabilidad social de las respuestas, tanto de hombres como mujeres, en un tema tan delicado como la violencia en pareja (De las Cuevas y González de Rivera, 1992), sin olvidar la culpabilización, negación o minimización de las conductas violentas (Scott y Straus, 2007) donde cuestiones como la satisfacción con la relación (Marshall, Panuzio, Makin-Byrd, Taft y Holtzworth-Munroe, 2011) o aspectos de género pueden influir en la interpretación de conductas ambiguas como violentas (Ryan y Mohr, 2005) o en la memoria de hombres y mujeres para recordar actos violentos (Kimmel, 2002). 
No obstante que esto puede ser algo relativamente sencillo de corregir, al menos en el caso de la deseabilidad social ya que existen numerosos instrumentos con versiones largas o cortas dirigidas a evaluar esta variable y que pueden ser fácilmente incluidas en los cuestionarios, sigue siendo un tema pendiente para las futuras investigaciones.

Ahora bien, el 30\% de los estudios revisados se realizó en la capital del país, pero se puede observar que gradualmente se llevan a cabo estudios en el interior de la República Mexicana. Y, como es esperable, las facultades donde se llevaron a cabo gran parte de los estudios revisados fueron Medicina, Psicología o Ciencias Sociales y el 95\% de los estudios provinieron de muestras de instituciones públicas.

A este respecto es pertinente llamar la atención sobre la necesidad de ampliar el radio de observación del fenómeno a otras facultades y, sin duda, sería muy necesario conocer más sobre el estado del fenómeno en las universidades privadas, como hicieron Adams y Cervantes (2012), al comprobar que la violencia en pareja no es algo privativo de un estrato socio-económico determinado.

Añadido a ello, aunque "una muestra no aleatoria es preferible a un vacío de información" (Argibay, 2009, p. 19) y cada una de ellas contribuye al entendimiento de la violencia interpersonal (McHugh, Livingston y Ford, 2005), esto no implica omitir las críticas a este tipo de muestreo. En ese sentido, es muy importante ser muy cautos al considerar las características de las muestras para la interpretación de los hallazgos obtenidos, pues variables demográficas como raza, estatus socioeconómico, localización geográfica o la diferencia entre el estilo o condiciones de vida asociados a un contexto urbano o rural pueden derivar en diferentes resultados pese a que se trate de estudios con similares objetivos y características (Miller, 2011). 
Por lo tanto, en aras de alejarse del "centralismo de las instituciones y las organizaciones no gubernamentales que trabajan temas de violencia de género" (Fonseca y Quintero, 2008, p. 176) y, agregaríamos, también de violencia de pareja con un enfoque bidireccional, lo ideal sería una selección probabilística y un tamaño y características de la muestra que permitan la generalización y réplica de los resultados obtenidos con datos provenientes de distintos Estados del país en aras de construir y fortalecer corpus empíricos tanto nacional como estatales.

En cuanto a los aspectos de contenido de los estudios revisados, es muy necesario hacer hincapié en que los resultados de los trabajos incluidos coinciden en señalar la presencia de violencia en las parejas jóvenes mexicanas.

Así, entre los hallazgos más relevantes se puede mencionar que la violencia psicológica parece ser la más frecuente (Ramirez y Smithey, 2008) sin dejar de mencionar la posible coexistencia de varios tipos de violencias (Olvera et al., 2012) o la implicación de ambos sexos como perpetradores -lo que no significa equiparar, claro está, las consecuencias sufridas por la violencia- (Rangel y García, 2010; Straus, 2004; Straus y Ramírez, 2007).

Además, se ha apuntado una suerte de normalización o minimización del uso de la violencia (Sánchez y Solís, 2007), lo que no es baladí ya que igualmente ha sido sugerido por estudios extranjeros al advertir que la presencia de una agresión y su respuesta, aunque sea en forma de represalia o autodefensa, a largo plazo podría favorecer una cultura de la violencia en algunas parejas (Olsen, Parra y Bennett, 2010). De esta forma, las generaciones más jóvenes también podrían estar aprendiendo interacciones que mantienen o estimulan la violencia, fomentando las agresiones como medio válido de comunicación o solución de conflictos; algo que podría traducirse, por 
ejemplo, en escaladas de agresiones que inician por lo verbal y terminan en agresiones físicas (Sears, Byers y Price, 2007).

Por otro lado, sólo el 10\% de los trabajos ampliaron su panorama de observación al incluir algunas manifestaciones violentas "nuevas" que pueden ser ejercidas por ambos sexos; tal es el caso de Sánchez y Solís (2009) y Olvera et al. (2012) que estudiaron el control a través de teléfonos celulares. No obstante, esta es una línea de investigación que necesita ser desarrollada, pues los avances tecnológicos y la popularidad del uso de los medios asociados a ello precisan de mayor atención por parte de futuras investigaciones.

Asimismo, es preciso llamar la atención sobre la pervivencia de algunos aspectos de socialización de género presentes en la violencia de pareja (Sánchez y Solís, 2007) que, concretamente, pudieran estar mediando en la perpetración de agresiones (Oliva et al., 2012), concretamente en aquellas de naturaleza sexual por parte de los hombres (Saldívar et al., 2008), además que pueden estar mediando en la identificación de las agresiones (Rangel y García, 2010) o la tolerancia de las mismas por parte de las mujeres (Fonseca y Quintero, 2008).

Por lo tanto, las diversificaciones de las relaciones de pareja de los jóvenes adultos mexicanos, aunque lleven la etiqueta de "noviazgo", así como las formas de la violencia, seguirán demandan igualmente modificaciones en el acercamiento del estudio de los fenómenos que ocurren en su seno. Se trata de cuestiones que, sin duda, requerirán de subsecuentes investigaciones que pueden apuntar nuevos derroteros en la forma de concebir el fenómeno y sus manifestaciones. Algo que a la postre podría incidir en la continuación o modificación de las acciones preventivas llevadas a cabo hasta ahora. 
Por último, pero no menos importante, es preciso señalar que sólo dos estudios se interesaron por los posibles factores protectores ante la violencia de pareja: el nivel educativo, en el trabajo de Ramírez y Núñez (2010), o una alta autoestima, según la investigación de Torres et al. (2011). Al respecto es preciso señalar que la lectura del fenómeno desde un reposicionamiento hacia la psicología positiva, es decir aquel “estudio científico del funcionamiento humano óptimo" (Seligman, 1999, citado por Salanova y López-Zafra, 2011, p. 340) con un enfoque que busque incrementar la salud y el bienestar (Lupano y Castro, 2010) a través de la identificación de habilidades, recursos como la resiliencia, rasgos positivos o factores protectores (Hervás, 2009), es una línea de investigación que podría proveer de mayor eficacia y eficiencia a los programas de prevención sobre esta problemática.

\section{Comentarios finales}

El estudio de la violencia en el noviazgo de universitarios mexicanos ha experimentado un gran crecimiento, sobre todo en los últimos años, formando gradualmente un corpus empírico necesario y útil para la prevención e intervención de esta problemática que, a la luz de los estudios revisados, no es privativa de un sexo ni ajena a alumnado universitario en México.

Por ello, entre otras sugerencias derivadas de esta revisión narrativa, se puede destacar la gran necesidad de diseños mixtos o longitudinales en ulteriores estudios. Además de la pertinencia de muestras representativas y seleccionadas no intencionalmente, en ese sentido el mayor tamaño y diversificación en las características de las muestras podría proveer de mayor profundidad en la descripción y conocimiento del fenómeno. Asimismo, sería muy conveniente la implementación de instrumentos validados que permitan la contrastación de los resultados obtenidos. 
Sin embargo, el error teórico y metodológico que comporta seguir interpretando e investigando un fenómeno diádico, como la violencia, desde una perspectiva individual es algo que no debe perderse de vista. Por lo que en futuras investigaciones la inclusión de parejas sería imprescindible para trasladar el tratamiento teórico y metodológico del objeto de estudio desde lo individual a su subsistema más próximo, la pareja; lo que sin lugar a dudas supondría un cambio epistemológico muy importante.

Sin detrimento de lo anterior, es muy necesario hacer énfasis en la inclusión del estudio de "las nuevas violencias" en la pareja, concretamente aquellas asociadas a las nuevas tecnologías, sin omitir la necesidad de ampliar el margen de estudio de la violencia de pareja entre los jóvenes adultos mexicanos y mexicanas hacia las nuevas formas de relaciones interpersonales.

Por último, es indispensable señalar que entre las principales limitaciones de este trabajo se encuentran los sesgos propios de una revisión narrativa entre los que destacan, siguiendo a Lozano (2005), que las apreciaciones críticas realizadas en este artículo han tenido criterios variables que han dependido del autor del presente artículo; no se evaluó la calidad de los estudios primarios, tampoco se sintetizó cuantitativamente la información obtenida, ni se discutieron las fuentes de error como los sesgos de publicación.

\section{Referencias}

Adams, J., \& Cervantes, L. (2012). Violencia pasiva en mujeres universitarias. Un estudio exploratorio de las causas del deterioro de la autoestima. Psicología y Salud, 22(1), 133-139.

Anderson, K. L. (2005). Theorizing gender in intimate partner violence research. Sex Roles, 52, 853-865. doi:10.1007/s11199-005-4204-x 
Argibay, J. C. (2009). Muestra en investigación cuantitativa. Revista Subjetividad y Procesos Cognitivos, 13, 13-29.

Casanova, C. L., \& Russi, I. M. (2007). Violencia en el noviazgo y su influencia en el rendimiento académico de los estudiantes universitarios. Acalán, Revista de la Universidad Autónoma del Carmen, 49, 12-15.

Castro, R. y Casique, I. (2010). Violencia en el noviazgo entre los jóvenes mexicanos. Cuernavaca: UNAM.

Castro, R., \& F. Riquer (2003). La investigación sobre la violencia contra las mujeres en América Latina: entre el empirismo ciego y la teoría sin datos. Cadernos de Saúde Pública, Río de Janeiro, 19(1), 35-146.

Castro, R., \& Riquer, F. (2004). Marco conceptual. En busca de nuevas direcciones hacia las cuales mirar. En R. Castro, F. Riquer, F. y M. A. Medina (Coords.). Violencia de género en las parejas mexicanas. Resultados de la Encuesta Nacional sobre la Dinámica de las Relaciones en los Hogares 2003 (pp. 1327). México, D.F.: Instituto Nacional de las Mujeres.

Cruz, Y., \& Cruz, A. K. (2008). La educación superior en México. Tendencias y desafíos. Revista da Avaliação da Educação Superior, 13(2), 293-311

De las Cuevas, C., \& González de Rivera, J. (1992). Autoinformes y respuestas sesgadas. Anales de Psiquiatría, 8(9), 362-366.

Dixon, L., Archer, J., \& Graham-Kevan, N. (2012). Perpetrator programmes for partner violence: Are they based on ideology or evidence? Legal and Criminological Psychology, 17, 196-215. doi:10.1111/j.2044-8333.2011.02029.x 
Dixon, L., \& Graham-Kevan, N. (2011). Understanding the nature and etiology of intimate partner violence and implications for practice and policy. Clinical Psychology Review, 31, 1145-1155. doi:10.1016/j.cpr.2011.07.00

Dutton, D. G. (1994). Patriarchy and wife assault: the ecological fallacy. Violence and Victims, 9(2), 167-182.

Escoto, Y., González, M., Muñoz, A. y Salomon, Y. (2007). Violencia en el noviazgo adolescente. Revista Internacional de Psicología, 8(2), 1-33.

Fonseca, C., \& Quintero, M. L. (2008). Igualdad de género entre jóvenes universitarios de la Universidad Autónoma del Estado de Hidalgo en la ciudad de Pachuca. En C. Fonseca y M. L. Quintero (Coord.), Temas emergentes en los estudios de género (pp. 173-183). México: Editorial Porrúa.

García, M., \& Romero, A. (2011). Coerción sexual en el noviazgo: Caracterización de jóvenes universitarios de la ciudad de Pachuca. En XXXVIII Congreso Nacional del Consejo Nacional para la Enseñanza e Investigación en Psicología, 13-15 de abril de 2011. México D.F.: UNAM. Recuperado el 12 $\begin{array}{llll}\text { de } & \text { septiembre } & \text { de } & 2012\end{array}$ http://www.uaeh.edu.mx/investigacion/icsa/LI_EvaluInter/Meli_Garcia/3.pdf

González, J, Hernández, A., \& Garza, R. I. (2010). Modelo predictivo de las interacciones violentas en parejas jóvenes y prejuicios de género asociados. Revista Científica Electrónica de Psicología, 10, 224-239. Recuperado el 3 de noviembre de 2012 de http://dgsa.uaeh.edu.mx/revista/psicologia/IMG/pdf/14___No._10.pdf

Hervás, G. (2009). Psicología positiva: una introducción. Revista Interuniversitaria de Formación del Profesorado, 66(23), 23-41. 
Huston, T. L., \& Robins, E. (1982). Conceptual and methodological issues in studying close relationships. Journal of Marriage and Family, 44(4), 901-925. doi:10.1017/S0033291700020882

Instituto Mexicano de la Juventud. (2008). Encuesta nacional de violencia en las relaciones de noviazgo 2007. México, D.F.

Kanin, E. J. (1957). Male aggression in dating-courting relations. American Journal of Sociology, 63, 197-204. doi:10.1086/222177

Kenny, D. A. y Cook, W. (1999). Partner effects in relationship research: conceptual issues, analytic difficulties, and illustrations. Personal Relationships, 6, 433448. doi:10.1111/j.1475-6811.1999.tb00202.x

Kimmel, M. S. (2002). "Gender symmetry" in domestic violence. A substantive and methodological research review. Violence Against Women, 8(11), 1332-1363. doi: $10.1177 / 107780102237407$

Lorber, M. F., \& O'Leary, K. D. (2012). Stability, change, and informant variance in newlyweds' physical aggression: individual and dyadic processes. Aggressive Behavior, 38, 1-15. doi:10.1002/ab.20414

Lozano, J. M. (2005). De patos, gansos y cisnes. Revisiones narrativas, revisiones sistemáticas y meta-análisis de la literatura. Acta Médica Colombiana, 30(1), $1-3$.

Lumbreras, I., Moctezuma, M. G., Dosamantes, L. D., Medina, M. A., Cervantes, M, López, M. R., \& Méndez, P. (2009). Estilo de vida y riesgos para la salud en estudiantes universitarios: hallazgos para la prevención. Revista Digital Universitaria, 10(2), 1-14. Recuperado el 1 de octubre de 2012 de http://www.revista.unam.mx/vol.10/num2/art12/int12.htm 
Lupano, M. L., \& y Castro, A. (2010). Psicología positiva, análisis desde su surgimiento. Ciencias Psicológicas, 4(1), 43-56.

Makepeace, J. M. (1981). Courtship violence among college students. Family Relations, 30, 90-102.

Marshall, A. D., Panuzio, J., Makin-Byrd, K. N., Taft, C. T., \& Holtzworth-Munroe, A. (2011). A multilevel examination of interpartner intimate partner violence and psychological aggression reporting concordance. Behavior Therapy, 42, 364377. doi:10.1016/j.beth.2010.09.003

McHugh, M. C., Livingston, N. A., \& Ford, A. (2005). A postmodern approach to women's use of violence: developing multiple and complex conceptualizations. Psychology of Women Quarterly, 29, 323-336. doi:10.1111/j.1471-6402.2005.00226.x

Melander, L. A., Noel, H., \& Tyler, K. A. (2010). Bidirectional, unidirectional, and nonviolence: a comparison of the predictors among partnered young adults. Violence and Victims, 25(5), 617-630. doi:10.1891/0886-6708.25.5.617

Miller, L. M. (2011). Physical abuse in a college setting: a study of perceptions and participation in abusive dating relationships. Journal of Family Violence, 26(1), 71-80. doi:10.1007/s10896-010-9344-2

Mora, J., Natera, G., Tiburcio, M., \& Juárez, F. (2008). Propiedades psicométricas de la escala de tácticas de conflicto (CTS2) en mujeres mexicanas. Revista Mexicana de Psicología, 25, 107-117.

Oliva, L., González, M. P., Yedra, L. R., Rivera, E. A., \& León, D. (2012). Agresión y manifestaciones violentas en el noviazgo en universitarios. Revista Psicología.com, 16(1), 1-12. Recuperado el 1 de noviembre de 2012 de 
http://www.psiquiatria.com/revistas/index.php/psicologiacom/article/viewFile/

\section{$\underline{1370 / 1246 /}$}

Olsen, J. P., Parra, G. R. y Bennett, S. A. (2010). Predicting violence in romantic relationships during adolescence and emerging adulthood: A critical review of the mechanisms by which familial and peer influences operate. Clinical Psychology Review, 30, 411-422. doi:10.1016/j.cpr.2010.02.002

Olvera, J. A., Arias, J., \& Amador, R. (2012). Tipos de violencia en el noviazgo: Estudiantes universitarias de la UAEM, Zumpango. Revista Electrónica de Psicología Iztacala, 15(1), 150-171. Recuperado el 3 de diciembre de 2012 de http://www.revistas.unam.mx/index.php/repi/article/view/30908

Perry, D. G., \& Pauletti, R. E. (2011). Gender and adolescent development. Journal of Research on Adolescence, 21, 61-74. doi:10.1111/j.1532-7795.2010.00715.x

Ramírez, C. A., \& Núñez D. A. (2010). Violencia en la relación de noviazgo en jóvenes universitarios: un estudio exploratorio. Enseñanza e Investigación en Psicología, 15(2), 273-283.

Ramírez, I. L., \& Smithey, M. (2008). Intimate partner violence victimization among Mexican university students: A descriptive study and analysis of demographic factors. Contemporary Issues in Criminology and the Social Sciences, 2(4), $34-53$.

Rangel, Y. Y., \& García, M. (2010). Influencia del rol de género en las formas de canalización de violencia en estudiantes universitarios mexicanos. En XI Congreso Virtual de Psiquiatría Interpsiquis 2010, 1-28 de febrero de 2010. España: Psiquiatria.com. 
Rodríguez-Franco, L., López-Cepero, J., Rodríguez, F. J., Bringas, C., Antuña, M. A., \& Estrada, C. (2010). Validación del Cuestionario de Violencia entre Novios (CUVINO) en jóvenes hispanohablantes: Análisis de resultados en España, México y Argentina. Anuario de Psicología Clínica y de la Salud, 6, 45-52.

Romo, J. M. (2008). Estudiantes universitarios y sus relaciones de pareja. De sus experiencias y proyectos de vida. Revista Mexicana de Investigación Educativa, 13(38), 801-823.

Rojas-Solís, J. L. (2011). Transformaciones socioculturales y aspectos de género: Algunas implicaciones para el estudio de violencia en pareja. Revista Electrónica de Psicología Iztacala, 14(3), 252-272. Recuperado el 1 de octubre de 2012 de http://www.revistas.unam.mx/index.php/repi/article/view/27655

Ruiz, M. A., Pardo, A. y San Martín, R. (2010). Modelos de Ecuaciones Estructurales. Papeles del Psicólogo, 31(1), 34-45.

Ryan, K. M., \& Mohr, S. (2005). Gender differences in playful aggression during courtship in college students. Sex Roles, 53, 591-601. doi:10.1007/s11199005-7144-6

Salanova, M., \& López-Zafra, E. (2011). Introducción: Psicología Social y Psicología Positiva. Revista de Psicología Social, 26(3), 339-343.

Saldívar, G., Ramos, L., \& Romero, M. P. (2008). ¿Qué es la coerción sexual? Significado, tácticas e interpretación en jóvenes universitarios de la Ciudad de México. Salud Mental, 31(1), 45-51. 
Saldívar, G., Ramos, L., \& Saltijeral, M. T. (2007). La aceptación de la violencia y los mitos de violación en estudiantes universitarios: Diferencias por sexo, edad y carrera. Revista de la Facultad de Medicina de la UNAM, 50(2), 71-75.

Saldívar, G., \& Romero, M. P. (2009). Reconocimiento y uso de tácticas de coerción sexual en hombres y mujeres en el contexto de relaciones heterosexuales. Un estudio en estudiantes universitarios. Salud Mental, 32(6), 487-494.

Sánchez, A. R., \& Solís, M. J. (2007). Estudiantes universitarios: percepción y vivencias de la sexualidad. En IX Congreso Nacional de Investigación Educativa, 5-9 de noviembre de 2007. Yucatán: COMIE. Recuperado el 6 de septiembre de 2012 de http://www.comie.org.mx/congreso/memoriaelectronica/v09/ponencias/at16/P $\underline{\text { RE1178980441.pdf }}$

Sánchez, A. R., \& Solís, M. J. (2009). Las huellas de la violencia en el noviazgo: estudiantes universitarios y trayectorias escolares de la FES Acatlán. En XI Congreso Nacional de Investigación Educativa, 21-25 de septiembre de 2009. Veracruz: COMIE. Recuperado el 3 de noviembre de 2012 de http://www.comie.org.mx/congreso/memoriaelectronica/v10/pdf/area_tematica _16/ponencias/0780-F.pdf

Scott, K., \& Straus, M. (2007). Denial, minimization, partner blaming, and intimate aggression in dating partners. Journal of Interpersonal Violence, 22(7), 851871. doi:10.1177/0886260507301227

Sears, H. A., Byers, E. S. y Price, A. L. (2007). The co-occurrence of adolescent boys' and girls' use of psychologically, physically, and sexually abusive behaviours 
in their dating relationships. Journal of Adolescence, 30, 487-504. doi:10.1016/j.adolescence.2006.05.002

Shorey, R. C., Cornelius, T. L., \& Bell, K. M. (2008). A critical review of theoretical frameworks for dating violence: Comparing the dating and marital fields. Aggression and Violent Behavior, 13, 185-194. doi:10.1016/j.avb.2008.03.003

Straus, M. A. (2004). Prevalence of violence against dating partners by male and female university students worldwide. Violence Against Women, 10(7), 790-811. doi:10.1177/1077801204265552

Straus, M. A., Hamby, S. L., Boney-McCoy, S., \& Sugarman, D. B. (1996). The Revised Conflict Tactics Scales (CTS2): Development and preliminary psychometric data. Journal of Family Issues, 17(3), 283-316. doi:10.1177/019251396017003001

Straus, M. A., \& Ramírez, I. L. (2007). Gender symmetry in prevalence, severity, and chronicity of physical aggression against dating partners by university students in Mexico and USA. Journal of Aggressive Behavior, 33(4), 281-290. doi:10.1002/ab.20199

Thompson, E. H. (1991). The maleness of violence in dating relationships: an appraisal of stereotypes. Sex Roles, 24, 261-278. doi:10.1007/BF00288301

Torres, M. E., Carreón, J. M., Sánchez, B. E., Bernal, L. I., \& Peña, E. (2011). Factores asociados a la violencia de pareja, en universitarias del área de la salud del estado de Guerrero, México. En Memorias del V Congreso Internacional de Salud Mental y Adicciones, 6 y 7 de octubre de 2011. Querétaro: Universidad Autónoma de Querétaro. Recuperado el 3 de octubre de 2012 de 
http://www.uaq.mx/enfermeria/5Foro_Enfermeria/memorias/t1/oal/o_gnvio/ro gv 3.pdf

Trujano, P., Dorantes, J., \& Tovilla, V. (2009). Violencia en internet: nuevas víctimas, nuevos retos. Liberabit. Revista de Psicología, 15(1), 7-19.

Valencia, M. J., García, M. L., Lozano, M. M., \& Flores, T. (2012). Comportamientos de violencia en el noviazgo a nivel universitario. Revista Paraninfo Digital, 16. Recuperado el 1 de diciembre de 2012 de http://www.indexf.com/para/n16/040d.php

Villaseñor-Farías, M. (2005). Andar de novios. En B. Rasmussen y A. Hidalgo (Coords.), Investigaciones en salud de adolescentes II (pp. 213-221). Guadalajara: Instituto Mexicano del Seguro Social y Organización Panamericana de la Salud.

Vizzuetth, A., García, M., \& Guzmán, R. (2010). Expectativas sobre la relación de amigovios, free y novios en jóvenes adultos. En S. Rivera-Aragón, R. DíazLoving, I. Reyes-Lagunes, R. Sánchez Aragón y L. M. Cruz Martínez (Eds.), La Psicología Social en México, Vol. 13 (pp. 223-230). México: AMEPSO y UNAM.

Weiss, E. (2012). Los estudiantes como jóvenes. El proceso de subjetivación. Perfiles Educativos, 34(135), 134-148.

Wolfe, D. A., Scott, K. L., \& Crooks, C. V. (2005). Abuse and violence in adolescent girls' dating relationships. En D. J. Bell-Dolan, S. L. Foster y E. J. Mash (Eds.), Handbook of Behavioral and Emotional Problems in Girls (pp. 381414). New York: Plenum Publishers. 
Este texto está protegido por una licencia Creative Commons

$$
\underline{4.0} \text {. }
$$

Usted es libre para Compartir — copiar y redistribuir el material en cualquier medio o formato - y Adaptar el documen- to - remezclar, transformar y crear a partir del material- para cualquier propósito, incluso comercialmente, siempre que cumpla la condición de:

Atribución: Usted debe reconocer el crédito de una obra de manera adecuada, proporcionar un enlace a la licencia, e in- dicar si se han realizado cambios. Puede hacerlo en cualquier forma razonable, pero no de forma tal que sugiera que tie- ne el apoyo del licenciante o lo recibe por el uso que hace.

$\underline{\text { Resumendelicencia - Textocompletodelalicencia }}$ 